\title{
Thickness Dependency in Dielectric Breakdown Strength of Biaxially Oriented Polypropylene-Silica Nanocomposite Films
}

\author{
H. Ranta, I. Rytöluoto and K. Lahti \\ Tampere University of Technology, Department of Electrical Engineering \\ Tampere, Finland
}

\begin{abstract}
The power law relationship has been used to some extent in order to compare dielectric breakdown strength results between materials of varying thickness. However, especially in case of relatively new materials such as nanocomposites, it can be questioned whether the measured results actually behave accordingly to the power law and to what extent - most importantly, can the power law be used to predict properties of thinner films than those actually measured. This paper addresses the problem in case of biaxially oriented PPsilica nanocomposite films of different thickness, the breakdown results of which are compared and fitted to the power law relationship.
\end{abstract}

\section{Introduction}

The dielectric breakdown strength of polymer materials can be affected by the sample thickness through the so called volume effect which is commonly expressed by the power law relationship given by Eq. (1)

$$
E_{2}=E_{1}\left[d_{2} / d_{1}\right]^{-n}
$$

where $E_{2}$ is the breakdown strength at thickness $d_{2}$ (15 $\mu \mathrm{m}), E_{l}$ is the measured breakdown strength of the film whose thickness is $d_{l}$ and $n$ is a positive constant whose value depends on the material in question. For instance, a value of 0.4 has been used in previous publications related to polypropylene (PP) and PP-based composite materials [1], [2], [3].

The power law relationship, where applicable, can be used to compare breakdown performance of polymer materials with different average thicknesses. In experimental laboratory-stage materials such as polymer nanocomposites, keeping e.g. a film extrusion process constant for materials with slightly different recipes can easily cause this kind of thickness variation in the final material. On the other hand, it may be essential to keep the processing constant in order to exclude its other effects from the results. In addition, the overall quality and evenness of experimental laboratory-processed polymer materials can be inferior compared to products of well-optimized industrial scale processing.

However, a particular problem of using the thickness correction with experimental and new materials is that in order to confirm the parameters such as $n$ for a given material, one might need considerably more breakdown data points from samples of varying thickness than are practically available, due to e.g. restrictions in material amounts. The purpose of this paper is to discuss the possible applicability of the power law relationship in the case of PP-silica nanocomposites which have been studied extensively by the authors' research group, and also to shed light on this problem in general.

\section{Experimental}

\subsection{Studied materials}

The materials studied were processed at the Technical Research Center of Finland (VTT). The base polypropylene was capacitor-grade PP supplied by Borealis Polymers N.V., and the silica filler was Evonik Degussa GmBH's Aeroxide LE2 (primary particle size $12 \mathrm{~nm}$, surface area $200 \mathrm{~m}^{2} / \mathrm{g}$ ). Content of the silica filler was approximately 4.5 wt- $\%$. Powder-form polypropylene was first compounded into pellets with silica, using a twin screw compounder with high shear screw geometry. These pellets were extruded into cast film of roughly $100 \times 0.5 \mathrm{~mm}$ dimensions with minimal machine direction orientation. Processing was kept the same for both PP-silica and the pure reference PP; a more detailed description can be found e.g. in [1] Biaxial orientation (simultaneous, $162^{\circ} \mathrm{C}$ temperature) was performed for square samples cut from the cast films, using Brückner KARO IV sheet stretching machine. Two separate thickness classes of films were produced: the thinner sheets were stretched with 5.6x5.6 draw ratio, while for the thicker ones the ratio was either 2.6 (PP-silica and ref. PP) or 2.4 (additional, ref. PP only). Resulting breakdown sample thickness ranges were $11 \ldots 23 \mu \mathrm{m}, 37 \ldots 75 \mu \mathrm{m}$ and $50 \ldots 92 \mu \mathrm{m}$, respectively. All sample thicknesses were measured as 5-measurement averages from the breakdown test area using a Mitutoyo tabletop micrometer with a specified accuracy of $\pm 1 \mu \mathrm{m}$. Zero calibration of the micrometer was confirmed by additional intermediate measurements, in order to guarantee best possible accuracy.

\subsection{Measurement of dielectric breakdown strength}

Short-term dielectric breakdown strength tests with ramping ac and $\mathrm{dc}$ voltages were conducted in room temperature $\left(20 \pm 3{ }^{\circ} \mathrm{C}\right)$, using cylindrical approx. $1 \mathrm{~cm}^{2}$ steel electrodes with graphite plates smoothing the contact to the sample. The samples were immersed in transformer oil (Shell Diala DX) during test, keeping the immersion time minimal but constant in order to prevent swelling. The ramp speed was $400 \mathrm{~V} / \mathrm{s}$ for samples of 5.6 stretch ratio, $1000 \mathrm{~V} / \mathrm{s}$ for 2.6 ratio and $1500 \mathrm{~V} / \mathrm{s}$ for 2.4 ratio. Sample amount in one data set 
varied from 20 to 72 , depending on the film thickness, voltage form and material. The ref. PP thick films with 2.6 and 2.4 stretch ratios, for instance, had no observable difference in the results, so these were treated as a single set.

Most of the thin films' breakdown results have been published in [1] and [3] with power law correction applied. Results for the thicker films have not been published earlier. The PP-silica thin film dc breakdown data set also includes 32 additional samples which have been measured later on, adding to a total of 72 samples.

\section{Results}

\subsection{Breakdowns with ac voltage}

Figure 1 presents the complete data sets measured for the materials at ac voltage, plotted against the measured sample thickness. The corresponding power law fits (with least squares method) to this original data are also shown, along with R-squared values to give some indication about quality of fit. It can readily be seen from the overall low R-squared values that the qualities of fit obtained this way are extremely bad, owing
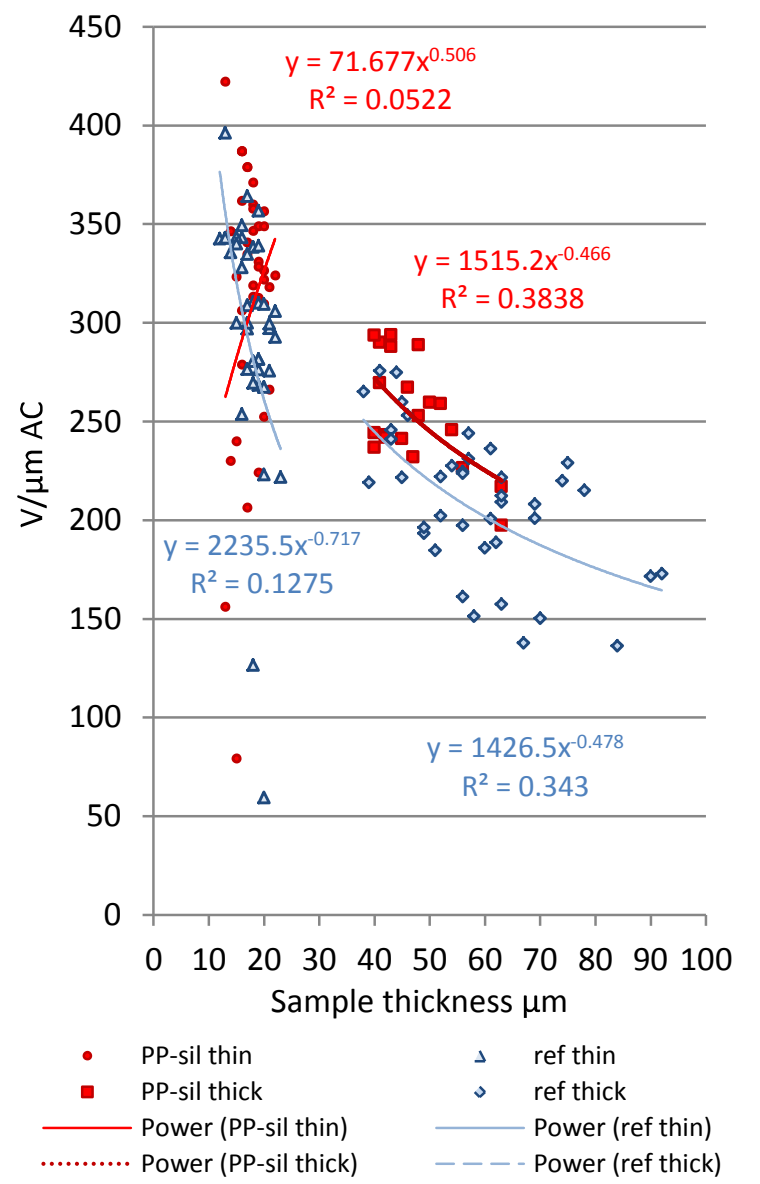

Fig. 1 - ac breakdown results as function of sample thickness and power law fittings to the individual result data sets, fitted separately for thin and thick films. mostly to large scattering of the results compared to amount of samples. Hence, little conclusions can be actually justified based on this data, which on the other hand is expectable considering the inherently stochastic nature of the breakdown process in polymers.

Most experimental $n$ values obtained seem to be positive, however, ranging from 0.466 to 0.717 . A strong exception of this is the thin PP-silica film set which has a negative $n$ of -0.506 . As can be seen, this is much affected by scattering at the lowest sample thicknesses (13 to $15 \mu \mathrm{m}$ ).

In order to control the effect of deviation, the results were arranged in groups by sample thickness, resulting in smaller subsets the size of which varied between 1 and 12 samples. For these, average breakdown strengths and standard deviations were calculated separately. The grouping was done at $1 \mu \mathrm{m}$ intervals for thin films and at $5 \mu \mathrm{m}$ for the thicker ones (due to greater variation in thickness, an average thickness within the range of each $5 \mu \mathrm{m}$ interval was calculated which was then plotted against the corresponding average breakdown strength.) Figure 2 presents the grouped results for the materials, showing group average and standard deviation (where
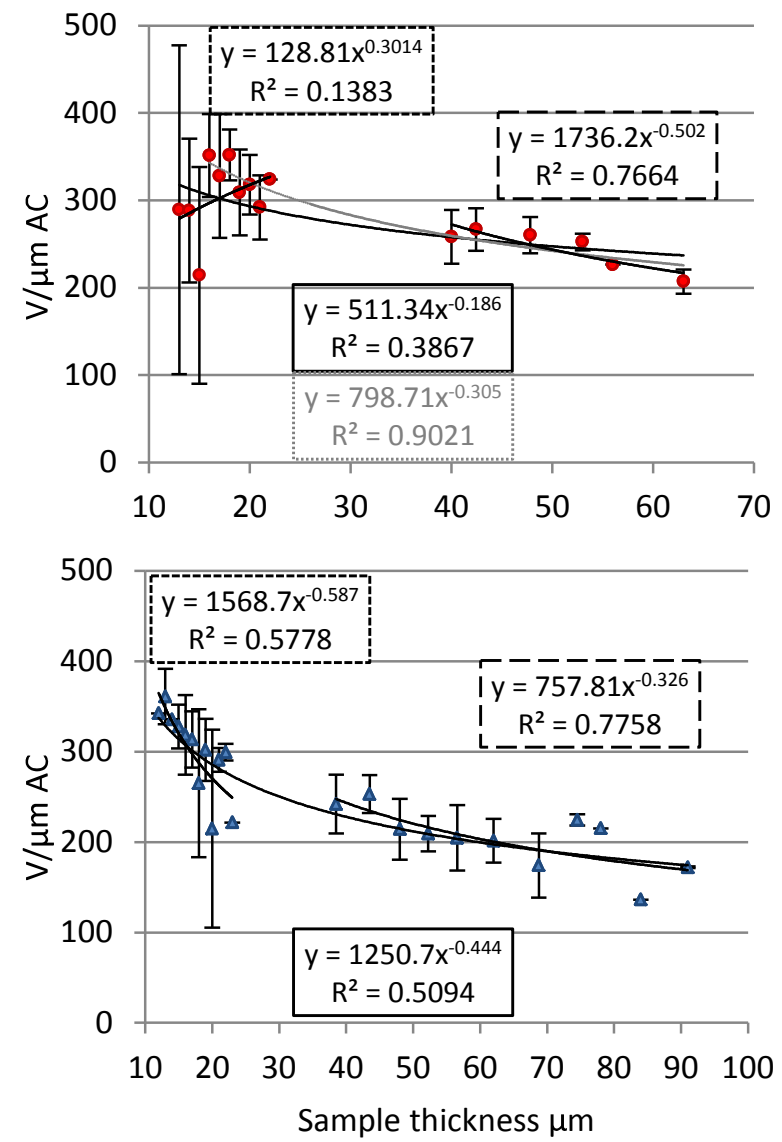

Fig. 2 - ac breakdown results plotted by subset averages for PP-silica (above) and ref. PP (below). Power law fits are done separately for thin and thick films (dashed black lines), and for thin and thick films combined (continuous black lines). Error bars represent standard deviation where applicable $(>1$ sample / subset). Grey dotted line is for PP-silica censored set which is missing the lowest three thickness groups. 


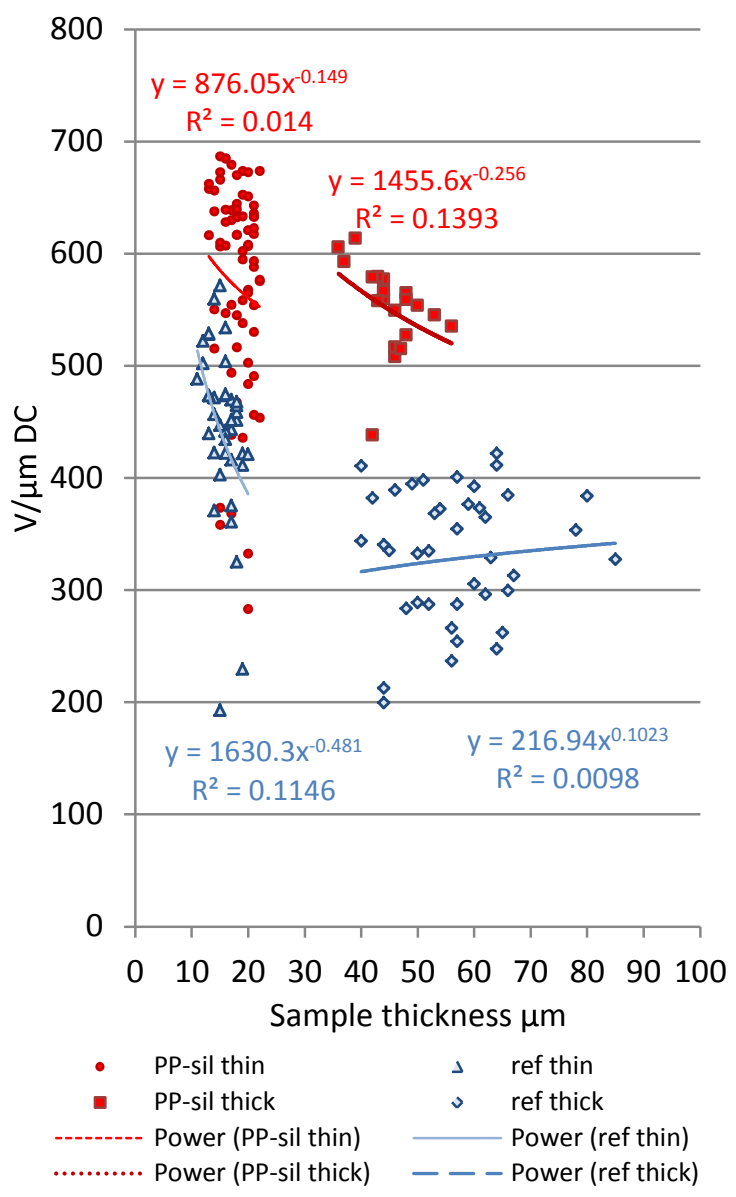

Fig. 3 - dc breakdown results as function of sample thickness and power law fittings to the individual result data sets, fitted separately for thin and thick films.

number of subset samples $>1$ ) for each thickness group. Power law fittings here have been calculated separately for thin and thick film averages, and for the combined data set containing both of these. For PP-silica, one additional fit was calculated which ignored the first three thickness groups (13 to $15 \mu \mathrm{m}$ ).

There appears to be slight improvement in the qualities of fit, although still low. Positive $n$ values now vary from 0.186 to 0.502 with PP-silica, whereas the thin films $n$ stays negative $(-0.3014)$. The effect of lowest three thickness groups is now even more clearly seen; if they would be omitted the quality of combined fit would improve considerably (see gray dotted curve). This is especially interesting given that the highest individual values were also within these groups, suggesting the existence of more than one failure mechanism. Ref. PP seems to follow the power law rather well, with $n$ increasing slightly towards lower thickness from 0.386 to 0.587 .

\subsection{Breakdowns with de voltage}

The dc voltage breakdowns are presented in Figures 3 and 4 showing the complete data sets and grouped averages, respectively. The $n$ values from direct data set
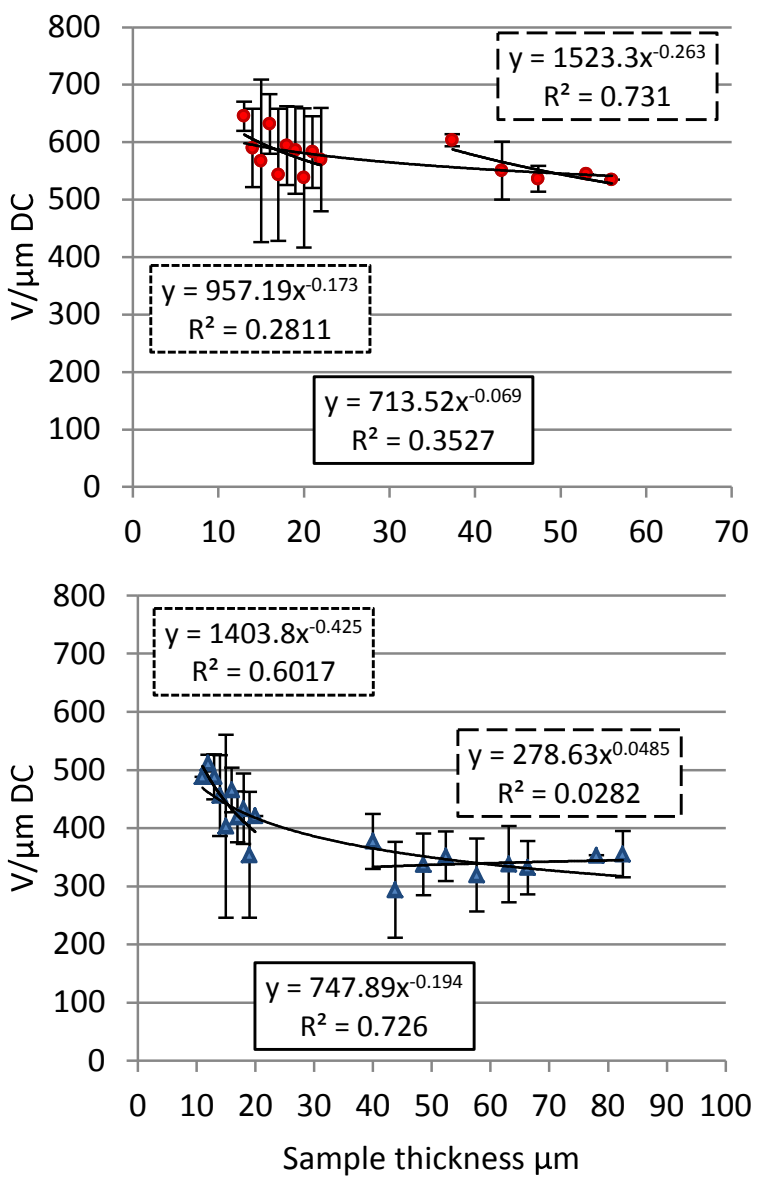

Fig. 4 - dc breakdown results plotted by subset averages for PP-silica (above) and ref. PP (below). Power law fits are done separately for thin and thick films(dashed black lines), and for thin and thick films combined (continuous black lines). Error bars represent standard deviation ( $>1$ sample / subset).

fits are 0.149 and 0.256 for thin and thick PP-silica, respectively, and 0.481 for thin PP ref., whereas thick PP ref. now shows a weakly negative $n$ of 0.1023 .

The situation is not much altered by grouping of the results, Fig. 4., although the quality of fits is again slightly improved. What is perhaps an interesting observation about PP-silica is that the combined power law fit curve is now relatively flat $(n=0.069)$, and the films of different thickness would seem to follow their individual power law relationships rather than a common one. This could be connected e.g. to effect of film orientation (different draw ratio).

\section{Discussion}

Because of the relatively small amount of data available, it is hard to say anything much conclusive about the results. It would seem, however, that while most of the studied data sets do not contradict the power law relationship, care should be taken in transferring the results outside the actual measured thickness range. Specifically the PP-silica material's ac breakdown behavior in the the lowest thickness range (negative $n$ value) raises questions, if one assumes there are 
Table 1 - Comparison of $n$ parameter values obtained from the different data sets.

\begin{tabular}{|c|c|c|c|c|}
\hline Data set & $\begin{array}{l}\text { ac } \\
n, \text { avg fit }\end{array}$ & $\begin{array}{l}\text { ac } \\
n \text { direct }\end{array}$ & $\begin{array}{l}\text { dc } \\
n \text {, avg fit }\end{array}$ & $\begin{array}{l}\mathrm{dc} \\
n \text { direct }\end{array}$ \\
\hline PP-sil thin & -0.3014 & -0.506 & 0.173 & 0.149 \\
\hline PP-sil thick & 0.502 & 0.466 & 0.263 & 0.256 \\
\hline PP-sil comb & 0.186 & & 0.069 & \\
\hline PP-sil cens. & 0.305 & & & \\
\hline PP ref thin & 0.587 & 0.717 & 0.425 & 0.481 \\
\hline PP ref thick & 0.326 & 0.478 & -0.0485 & -0.1023 \\
\hline PP ref comb. & 0.444 & & 0.194 & \\
\hline
\end{tabular}

physical mechanisms behind it instead of just coincidence (statistical variation).

Table 1 summarizes the power law $n$ parameters obtained from the experimental data sets. Generally, the $n$ parameter values obtained from individual thin films are slightly higher with reference PP than with PPsilica, whereas PP-silica gives higher values in the thicker films. At ac voltage $n$ is higher with both materials than with dc voltage. Direct fitting to the measured results made the analysis more susceptible to bad quality of fit, compared to the thickness-grouped average fitting.

Table 2 gives indication of the differences caused by power law correction, when the results are transferred to another thickness by it. Correcting to e.g. $15 \mu \mathrm{m}$ from the thin films (the thickness of which included this value) is likely to cause negligible error even in partly non-power law compliant cases, whereas further extrapolation (even to $9 \mu \mathrm{m}$ ) could cause a more significant error. As an extreme example, the measured average values for PP-silica thin and thick films at $\mathrm{dc}$ are nearly the same (578 and $552 \mathrm{~V} / \mu \mathrm{m})$; so an extrapolation to $15 \mu \mathrm{m}$ with $n=0.4$ would lead to a 243 $\mathrm{V} / \mu \mathrm{m}$ larger estimate made from the thicker film. On the other hand, when breakdown performance differences between two materials of different thickness are small, the proper application of power law may well be necessary in order to properly compare said materials.
Table 2 - Measured average breakdown strength values with their standard deviations at ac and $\mathrm{dc}$ voltages, and corresponding power law values corrected to different thickness as presented in earlier publications $\left(n=0.4, d_{2}=15\right.$ or $9 \mu \mathrm{m}$ ).

\begin{tabular}{|c|c|c|c|c|c|c|}
\hline \multirow[t]{2}{*}{ Data set } & \multirow{2}{*}{$\begin{array}{l}\mathrm{AC} \\
\mathrm{V} / \mu \mathrm{m} \\
\text { meas. }\end{array}$} & \multicolumn{2}{|c|}{$\begin{array}{l}\text { old correction } \\
(n=0.4)\end{array}$} & \multirow{2}{*}{$\begin{array}{l}\mathrm{DC} \\
\mathrm{V} / \boldsymbol{\mu m} \\
\text { meas. }\end{array}$} & \multicolumn{2}{|c|}{$\begin{array}{l}\text { old correction } \\
(n=0.4)\end{array}$} \\
\hline & & $15 \mu \mathrm{m}$ & $9 \mu \mathrm{m}$ & & $15 \mu \mathrm{m}$ & $9 \mu \mathrm{m}$ \\
\hline \multicolumn{7}{|l|}{ Thin films } \\
\hline \multirow{2}{*}{$\begin{array}{l}\text { PP-sil avg } \\
\text { stdev }\end{array}$} & 317 & 338 & 415 & 578 & 622 & 763 \\
\hline & 68 & 74 & 90 & 92 & 100 & 123 \\
\hline \multirow{2}{*}{$\begin{array}{l}\text { Ref. avg } \\
\text { stdev }\end{array}$} & 297 & 315 & 387 & 443 & 449 & 550 \\
\hline & 61 & 60 & 73 & 75 & 71 & 87 \\
\hline \multicolumn{7}{|l|}{ Thick films } \\
\hline \multirow{2}{*}{$\begin{array}{l}\text { PP-sil avg } \\
\text { stdev }\end{array}$} & 254 & 404 & 491 & 552 & 865 & 1049 \\
\hline & 27 & 36 & 42 & 39 & 74 & 69 \\
\hline \multirow{2}{*}{$\begin{array}{l}\text { Ref. avg } \\
\text { stdev }\end{array}$} & 209 & 400 & 437 & 333 & 589 & 716 \\
\hline & 36 & 79 & 61 & 58 & 102 & 131 \\
\hline
\end{tabular}

\section{References}

[1] M. Takala et al., "Dielectric Properties and Partial Discharge Endurance of Polypropylene-Silica Nanocomposite", IEEE Transactions on Dielectrics and Electrical Insulation, IEEE, Vol. 17, No. 4; August 2010, pp. 1259-1267.

[2] M. Takala et al., "Effect of Low Amount of Nanosilica on Dielectric Properties of Polypropylene" Solid Dielectrics (ICSD), 10th IEEE International Conference on, IEEE, Potsdam, Germany, 4-9 July 2010.

[3] H. Ranta and K. Kannus, " Effects of ElectricalThermal Aging on Short-Term Dielectric Strength of Biaxially Oriented Polypropylene-Silica Nanocomposite Films", Proceedings of the 22nd Nordic Insulation Symposium NORD-IS11, Tampere, Finland, 13-15 June, 2011 